\title{
PROPOSING THE PROMISCUOUS PROTEIN STRUCTURES IN JNK1 AND JNK3 FOR VIRTUAL SCREENING IN PURSUIT OF POTENTIAL LEADS
}

\author{
Sailapathi Ananthasri ${ }^{1+}$, Murugan Gopinath ${ }^{1 \dagger}$, Kanagasabai Somarathinam ${ }^{1}$, Seshan Gunalan ${ }^{1}$, \\ Rahul Jagadeesan ${ }^{1}$, Niyaz Yoosuf ${ }^{2}$, Sekar Kanagaraj ${ }^{3}$, Gugan Kothandan ${ }^{1 *}$
}

\begin{abstract}
${ }^{1}$ Biopolymer Modelling Laboratory, Centre of Advanced Study in Crystallography and Biophysics, Guindy Campus, University of Madras, Chennai - 600025, Tamilnadu, India.

${ }^{2}$ Department of Medicine, Solna Rheumatology unit, CMM L8:05 Karolinska university hospital, 171 76, Stockholm, Sweden, Email: niyaz.yoosuf@ki.se

${ }^{3}$ Laboratory for Structural Biology and Bio-computing, Department of Computational and Data Sciences, Indian Institute of Science, Bangalore - 560 012, India.

+ Authors with equal contribution

* Corresponding Author
\end{abstract}

\author{
AUTHOR INFORMATION SECTION \\ *Corresponding Author \\ Dr. Gugan Kothandan \\ Assistant professor, \\ CAS Crystallography and Biophysics \\ Guindy Campus, \\ University of Madras \\ Guindy, Chennai- 600025 \\ Phone: +91-9962312001 \\ Email:drgugank@gmail.com
}

AUTHOR CONTRIBUTIONS

Sailapathi Ananthasri and Murugan Gopinath have contributed equally to this work. 


\section{Loop Program:}

$\#$ ! /bin/bash

\# The path of the file containing the parameter files of the structures to be docked for d in /home/administrator/Desktop/adtrun/*/

do

\# To run autogrid on all the *.gpf files and also autodock on all the *.dpf files after an interval of 120 seconds

(cd "\$d"\&\& autogrid4 -p *.gpf -I grid.glg \&\& sleep $120 \& \&$ autodock4 -p *.dpf -I dock.dlg)\& done 